\title{
The GA4GH Phenopacket schema: A computable representation of clinical data for precision medicine
}

Julius O. B. Jacobsen[0000-0002-3265-1591]1,* Michael Baudis[0000-0002-9903-4248]2,3 Gareth S. Baynam[0000-0003-4920-9553]4,5,6 Jacques S. Beckmann[0000-0002-9741-1900]7 Sergi Beltran[0000-0002-2810-3445]8,9,10 Tiffany J. Callahan[0000-0002-8169-9049]11 Christopher G. Chute[0000-0001-5437-2545]12 Mélanie Courtot[0000-0002-9551-6370]13 Daniel Danis[0000-0003-0900-3411]14 Olivier Elemento[0000-0002-8061-9617]15 Robert R. Freimuth[0000-0002-9673-5612]16 Michael A. Gargano[0000-0002-2157-3591]14 Tudor Groza[0000-0003-2267-8333]17 Ada Hamosh[0000-0002-1780-5230]18 Nomi L. Harris[0000-0001-6315-3707]19 Rajaram Kaliyaperumal[0000-0002-1215-167X]20 Aly Khalifa[0000-0002-7084-1345]16 Peter M. Krawitz[0000-0002-3194-8625]21 Sebastian Köhler[0000-0002-5316-1399]22 Brian J. Laraway[0000-0002-0450-7074]11 Heikki Lehväslaiho[0000-0002-6263-1356]23 Kent C. Lloyd[0000-0002-5318-4144]24,25 Leslie Matalonga[0000-0003-0807-2570]8 Julie A. McMurry[0000-0002-9353-5498]11 Alejandro Metke-Jimenez[0000-0003-1068-0938]26 Christopher J. Mungall[0000-0002-6601-2165]19 Monica C. Munoz-Torres[0000-0001-8430-6039]11 Soichi Ogishima[0000-0001-8613-2562]27 Anastasios Papakonstantinou[0000-0003-4301-3859]8 Davide Piscia[0000-0002-0468-0408]8 Nikolas Pontikos[0000-0003-1782-4711]28,29 Núria Queralt-Rosinach[0000-0003-0169-8159]20 Marco Roos[0000-0002-8691-772X]20 Paul N. Schofield[0000-0002-5111-7263]30,31,32 Anastasios Siapos[0000-0001-6753-6764]33 Damian Smedley[0000-0002-5836-9850]1 Lindsay D. Smith[0000-0002-0603-4178]34,35 Robin Steinhaus[0000-0001-6613-4675]36,37 Jagadish Chandrabose Sundaramurthi[0000-0002-6670-9157]14 Emilia M. Swietlik[0000-0002-4095-8489]38,39,40 Sylvia Thun[0000-0002-3346-6806]41 Nicole A. Vasilevsky[0000-0001-5208-3432]42 Alex H. Wagner[0000-0002-2502-8961]43,44 Jeremy L. Warner[0000-0002-2851-7242]45 Claus Weiland[0000-0003-0351-6523]46 Melissa A. Haendel[0000-0001-9114-8737]11, * Peter N. Robinson[0000-0002-0736-9199]47,48, *

1. Queen Mary University of London, William Harvey Research Institute, London EC1M 6BQ, UK

2. University of Zurich, Department of Molecular Life Sciences, Zürich 8057, Switzerland

3. Swiss Institute of Bioinformatics, Computational Oncogenomics Group, Zürich 8057, CH

4. King Edward Memorial Hospital, Western Australian Register of Developmental Anomalies and Genetic Services of WA, Perth 6008, AU

5. University of Western Australia, Faculty of Health and Medical Sciences, Division of Paediatrics, Perth 6008, AU

6. Telethon Kids Institute, Genetic and Rare Diseases, Perth 6008, AU

7. University of Lausanne, Faculty of Biology and Medicine, Lausanne $\mathrm{CH}-1015$, Switzerland

8. CNAG-CRG, Centre for Genomic Regulation (CRG), The Barcelona Institute of Science and Technology, Bioinformatics Unit, Barcelona 8028, ES

9. Universitat Pompeu Fabra (UPF), Barcelona 8005, ES

10. Departament de Genètica, Microbiologia i Estadística, Facultat de Biologia, Universitat de Barcelona (UB), Barcelona 8028, ES

11. University of Colorado Anschutz Medical Campus, Center for Health Al, Aurora 80045, CO, USA

12. Johns Hopkins University, Schools of Medicine, Public Health, and Nursing, Baltimore 21287, MD, USA

13. European Molecular Biology Laboratory, European Bioinformatics Institute (EMBL-EBI), Hinxton CB10 1SD, UK

14. The Jackson Laboratory, Genomic Medicine, Farmington 6032, CT, USA

15. Weill Cornell Medicine, Caryl and Israel Englander Institute for Precision Medicine, New York 10021, NY, USA

16. Mayo Clinic, Department of Artificial Intelligence and Informatics, Rochester 55905, MN, USA

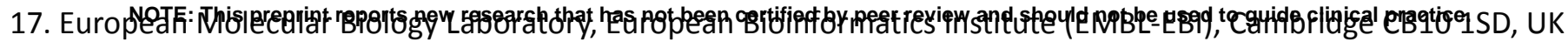
18. Johns Hopkins University, Department of Genetic Medicine, Baltimore 21287, MD, USA

19. Lawrence Berkeley National Laboratory, Environmental Genomics and Systems Biology, Berkeley 94720, CA, USA 
medRxiv preprint doi: https://doi.org/10.1101/2021.11.27.21266944; this version posted November 30, 2021. The copyright holder for this preprint (which was not certified by peer review) is the author/funder, who has granted medRxiv a license to display the preprint in perpetuity.

It is made available under a CC-BY 4.0 International license .

20. Leiden University Medical Center, Human Genetics, Leiden 2333 ZA, NL

21. University Hospital Bonn, Bonn, Germany, Institute for Genomic Statistics and Bioinformatics, Bonn 53113, DE

22. Ada Health $\mathrm{GmbH}$, Berlin 10178, DE

23. CSC - IT Center for Science, Sensitive Data Services, Espoo FI-02101, FI

24. UC Davis, Mouse Biology Program, Davis 95618, CA, USA

25. UC Davis School of Medicine, Surgery, Sacramento 95817, CA, USA

26. CSIRO, The Australian e-Health Research Centre, Herston 4029, AU

27. Tohoku University, INGEM, Sendai 980-8573, JP

28. University College London, Institute of Ophthalmology, London EC1V 9EL, UK

29. Moorfields Eye Hospital, Genetics Service, London EC1V 2PD, UK

30. University of Cambridge, Dept of Physiology, Development and Neuroscience, Cambridge CB2 3EG, UK

31. The Jackson Laboratory, Mammalian Genetics, Bar Harbor ME 04609, ME, USA

32. The Alan Turing Institute, London NW1 2DB, UK

33. Lifebit Biotech Ltd., London W86BD, UK

34. Ontario Institute for Cancer Research, Adaptive Oncology, Toronto M5G0A3, CA

35. Global Alliance for Genomics and Health, N/A, Toronto M5G0A3, CA

36. Berlin Institute of Health at Charité - Universitätsmedizin Berlin, Berlin 10178, DE

37. Charité - Universitätsmedizin Berlin, corporate member of Freie Universität Berlin and Humboldt-Universität zu Berlin, Institute of Medical Genetics and Human Genetics, Berlin 13353, DE

38. University of Cambridge, Medicine Department, Cambridge CB2 OQQ, UK

39. Addenbrooke's Hospital, Respiratory Medicine Department, Cambridge CB2 0QQ, UK

40. Royal Papworth Hospital, Cambridge Centre for Lung Infection, Cambridge CB2 OAY, UK

41. Berlin Institute of Health at Charité - Universitätsmedizin Berlin, Core Facility Digital Medicine and Interoperability, Berlin 10178, DE

42. University of Colorado, Anschutz Medical Campus, Center for Health Al, Aurora 80045, CO, USA

43. Nationwide Children's Hospital, The Steve and Cindy Rasmussen Institute for Genomic Medicine, Columbus 43215, OH, USA

44. The Ohio State University College of Medicine, Departments of Pediatrics and Biomedical Informatics, Columbus 43215, OH, USA

45. Vanderbilt University, Departments of Medicine and Biomedical Informatics, Nashville 37235, TN, USA

46. Senckenberg - Leibniz Institution for Biodiversity and Earth System Research, Data and Modelling Centre, Frankfurt/Main 60325, DE

47. The Jackson Laboratory, Genomic Medicine, Farmington 06032, CT, USA

48. University of Connecticut, Institute for Systems Genomics, Farmington 06032, CT, USA

\section{Consortial authors}

Myles Axton[0000-0002-8042-4131]1 Lawrence Babb[0000-0002-2455-2227]2 Cornelius F. Boerkoel[0000-0003-3097-241X]3 Bimal P. Chaudhari[0000-0002-0115-949X]4,5 Hui-Lin Chin[0000-0001-7431-6794]6,7 Michel Dumontier[0000-0003-4727-9435]8 David P. Hansen[0000-0002-2998-4563]9 Harry Hochheiser[0000-0001-8793-9982]10 Veronica A. Kinsler[0000-0001-6256-327X]11,12 Hanns Lochmüller[0000-0003-2324-8001]13,14,15 Alexander R. Mankovich[0000-0002-1258-4184]16 Gary I. Saunders[0000-0002-7468-0008]17 Panagiotis I. Sergouniotis[0000-0003-0986-4123]18 Rachel Thompson[0000-0002-6889-0121]13 Andreas Zankl[0000-0001-8612-1062]19,20,21

1. Wiley, Inc, Research, Hoboken 7030, NJ, USA

2. Broad Institute of MIT and Harvard, Cambridge 2142, MA, USA

3. University of British Columbia, Medical Genetics, Vancouver V6H3N1, CA

4. Nationwide Children's Hospital, The Steve and Cindy Rasmussen Institute for Genomic Medicine, Divisions of Neonatology, Genetics and Genomic Medicine, Columbus 43215, OH, USA

5. The Ohio State University College of Medicine, Department of Pediatrics, Columbus 43210, OH, USA 
medRxiv preprint doi: https://doi.org/10.1101/2021.11.27.21266944; this version posted November 30, 2021. The copyright holder for this preprint (which was not certified by peer review) is the author/funder, who has granted medRxiv a license to display the preprint in perpetuity.

It is made available under a CC-BY 4.0 International license .

6. Khoo Teck Puat-National University Children's Medical Institute, National University Hospital, Department of Paediatrics, Singapore 119074, Singapore

7. Women's Hospital of British Columbia, Provincial Medical Genetics, Vancouver V6H3N1, CA

8. Maastricht University, Institute of Data Science, Maastricht $6229 \mathrm{EN}, \mathrm{NL}$

9. CSIRO, Australian e-Health Research Centre, Brisbane 4027, AU

10. University of Pittsburgh, Biomedical Informatics, Pittsburgh 15206, PA, USA

11. Great Ormond St Hospital for Children, Paediatric Dermatology, London WC1N 3JH, UK

12. Francis Crick Institute, Mosaicism and Precision Medicine Laboratory, London NW1 1AT, UK

13. Children's Hospital of Eastern Ontario Research Institute, Molecular Biomedicine, Ottawa K1H 8L1, CA

14. University of Ottawa, Brain and Mind Research Institute, Department of Cellular and Molecular Medicine, Ottawa K1H 8M5, CA

15. The Ottawa Hospital, Neuromuscular Centre, Ottawa K1Y 4E9, CA

16. Philips Research North America, Precision Diagnosis \& Image-Guided Therapy, Cambridge 2141, MA, USA

17. ELIXIR, ELIXIR Hub, Cambridge CB10 1SD, UK

18. University of Manchester, Division of Evolution, Infection and Genomics, Manchester M13 9PT, UK

19. The University of Sydney, Faculty of Medicine and Health, Sydney 2006, AU

20. The Children's Hospital at Westmead, Department of Clinical Genetics, Westmead 2145, AU

21. Garvan Institute of Medical Research, Kinghorn Centre for Clinical Genomics and Bone Division, Darlinghurst 2010, AU

\section{Abstract}

Despite great strides in the development and wide acceptance of standards for exchanging structured information about genomic variants, there is no corresponding standard for exchanging phenotypic data, and this has impeded the sharing of phenotypic information for computational analysis. Here, we introduce the Global Alliance for Genomics and Health (GA4GH) Phenopacket schema, which supports exchange of computable longitudinal case-level phenotypic information for diagnosis and research of all types of disease including Mendelian and complex genetic diseases, cancer, and infectious diseases. To support translational research, diagnostics, and personalized healthcare, phenopackets are designed to be used across a comprehensive landscape of applications including biobanks, databases and registries, clinical information systems such as Electronic Health Records, genomic matchmaking, diagnostic laboratories, and computational tools. The Phenopacket schema is a freely available, community-driven standard that streamlines exchange and systematic use of phenotypic data and will facilitate sophisticated computational analysis of both clinical and genomic information to help improve our understanding of diseases and our ability to manage them.

\section{Introduction}

Despite the clinical importance of phenotypic features (signs, symptoms, laboratory and imaging findings, results of physiological tests, etc.), exchanging them in conjunction with genomic variation is often overlooked or even neglected. Although great progress has been made in exchange formats for sequence and variation data such as the Variant Call Format (VCF), ${ }^{1}$ complementary standards for phenotypic and other clinical data have lagged far behind. In the clinical domain, a significant amount of work has been dedicated to the development of computational phenotypes. ${ }^{2}$ Traditionally, these approaches have largely relied on rule-based methods and large sources of clinical data to identify cohorts of patients with or without a specific disease..$^{3-6}$ Unlike the aforementioned genomic standards, these approaches were not developed to model patient-level phenomena nor facilitate complex within and between patient comparisons. For example, one cannot readily compare an observed phenotypic profile against clinical databases as one can with genomic sequences using tools such as BLAST, ${ }^{2}$ partially because the lack of standards has impeded the development of algorithms and software for computational phenotype analysis. Another limitation is that phenotypic abnormalities of individuals are currently described in diverse places in multiple formats: ${ }^{7}$ scientific publications, biomedical databases, health records, patient health forums, ${ }^{8}$ and social media. ${ }^{9}$ The 
medRxiv preprint doi: https://doi.org/10.1101/2021.11.27.21266944; this version posted November 30, 2021. The copyright holder for this preprint (which was not certified by peer review) is the author/funder, who has granted medRxiv a license to display the preprint in perpetuity.

It is made available under a CC-BY 4.0 International license .

development of a reliable and practical standard for the documentation and exchange of clinical phenotypes is then critically necessary. A phenotype exchange standard is also timely for two reasons: firstly, there is increasing consensus about best practices for secure phenotypic data sharing and secondly, there have been significant advances in analytical methods to make effective use of computationally encoded phenotypic data. ${ }^{10}$

Deep phenotyping is a process whereby a multitude of morphological, biochemical, physiological, or behavioral attributes of each patient are collected by traditional clinical examinations and increasingly through advanced technologies such as imaging. ${ }^{11,12}$ Many diagnostic tools now leverage phenotype-driven differential diagnostic support on the basis of ontologies such as the Human Phenotype Ontology (HPO). ${ }^{13-30}$ Ontologies are systematic formal representations of knowledge that can be used to integrate and analyze large amounts of heterogeneous data by defining entities and concepts such as genetic variation, clinical findings, and diseases as well as the relationships between these concepts in a way that allows computational logical reasoning. ${ }^{10}$ Ontology-driven algorithms have been transformative for rare disease diagnosis. ${ }^{30,31}$ While the $\mathrm{HPO}^{32}$ offers over 16,000 terms to describe phenotypic abnormalities (symptoms, signs, laboratory abnormalities, behavioral manifestations, imaging findings, etc.), it does not itself provide a framework for exchanging information about the phenotypic abnormalities comparable to the way VCF provides a framework for exchanging information about all of the variants found in an exome or genome sequence. Such a framework would greatly facilitate precision medicine and precision public health. ${ }^{10}$

One challenge is that computational phenotype analysis is still poorly connected with the Electronic Health Record (EHR) and also that EHRs are not standardized across institutions or countries ${ }^{33}$. To enable precision medicine, standards and tools are needed to improve machine readable phenotypic characterization of patients beyond current standard EHR billing and clinical encounter data capture; EHR problem lists can only partly address this problem. To study disease trajectories and to optimize care plans for patients there is a need to identify correlations and changes in phenotypic characteristics with other data modalities such as imaging, patient-reported data including mobile health, 'omics data, etc. Standardization of phenotype data is necessary to gather together the sample sizes of hundreds of thousands of patients from widely distributed sources, as will be required to assess the clinical relevance of (rare) genetic variants in Mendelian and common diseases with sufficient statistical support. ${ }^{34-36}$ Many complex and infectious diseases would also benefit from a robust, computational representation of phenotypic characteristics and their temporal progression in association with genomic, other 'omics, environmental, mobile health data, etc. ${ }^{10}$ Further, increasingly detailed phenotypes are required to improve our understanding of how specific variants can predict patient phenotypic sub-groups, complications, progression rates, and/or response to therapy. Scalable deep phenotyping will be important for both rare and complex diseases within learning health systems. ${ }^{37,38}$

The Global Alliance for Genomics and Health (GA4GH) is developing a suite of coordinated standards for genomics for healthcare. ${ }^{39}$ The Phenopacket is a new GA4GH standard for sharing disease and phenotype information. To build the standard, requirements and specifications were established through a community effort. The standard underwent a rigorous peer review and product approval process and was consequently promoted as a GA4GH standard. Version 1 of the GA4GH standard was released in 2019 to elicit feedback from the community. Version 2 was developed on the basis of this feedback and is described here. A Phenopacket characterizes an individual person or biosample, linking that individual to detailed phenotypic descriptions, genetic information, diagnoses, and treatments (Figure 1). The Phenopacket schema enables comparison of sets of phenotypic attributes from individual patients. Such comparisons can aid in diagnosis and facilitate patient classification and stratification for identifying new diseases and treatments. ${ }^{10}$ The Phenopacket schema is designed to support interoperability between the people, organizations, and systems that comprise the worldwide effort to address human disease and biological understanding. These partners include clinical laboratories, authors, journals, clinicians, data repositories, patient registries, EHRs, and knowledge bases; the structure of the information in a phenopacket was designed for integration within these distributed contexts. Increasing the volume of computable data across a diversity of systems will support global computational disease analysis by integrating genotype, phenotype, and other multi-modal data for precision health applications. 
medRxiv preprint doi: https://doi.org/10.1101/2021.11.27.21266944; this version posted November 30, 2021. The copyright holder for this preprint (which was not certified by peer review) is the author/funder, who has granted medRxiv a license to display the preprint in perpetuity.

It is made available under a CC-BY 4.0 International license.

\section{Results}

The GA4GH Phenopacket Schema is a set of rules that specify the organization of data as a blueprint for constructing a phenopacket, which is a structured representation of an individual's medically relevant data, providing a computable case report of either a single medical encounter or a time course that can represent the entire medical history of an individual. It includes information such as age (which can be represented in multiple ways including ranges to protect privacy), sex, and gender as well as existing disease diagnoses. Almost all elements of a phenopacket are optional. A simple phenopacket contains only information about the individual and a list of phenotypic features and is sufficient for use cases of Mendelian disease genomic diagnostics. More comprehensive phenopackets contain data about biosamples and treatment associated with an individual patient along with phenotypic features and can be appropriate for use in clinical management beyond diagnosis, such as treatment selection.

\section{Schema overview}

Currently, a very wide range of ad hoc database schemas are used to represent clinical data for specific research projects, and numerous different ontologies are used to represent clinical entities; for instance, diseases can be represented by terms from ontologies and terminologies including Mondo, OMIM, Orphanet, NCIT, ICD, and many others. ${ }^{40}$ Therefore, the Phenopacket schema is intentionally flexible to facilitate wide adoption and to increase the value of the network of systems sharing phenopackets for computational use. The major goals for the Phenopacket schema include composability, traceability (data provenance), the FAIR principles (Findable, Accessible, Interoperable, and Reusable), and computability. ${ }^{41-44}$ Specifically, Phenopackets are designed to be both human and machine-interpretable, enabling computing operations and validation on the basis of defined relationships between diagnoses, lab measurements, and genotypic information; they also enable seamless transfer of data from a data source (e.g., a document describing the phenotypic information) to a data receiver (e.g., an application that receives and uses it). The standard supports several computational document formats such as JSON and YAML. Phenopackets require common and well-established ontologies, that is, logically defined hierarchies of terms that allow sophisticated algorithmic analysis over medically relevant abnormalities. ${ }^{10}$ The Phenopacket schema does not directly model -omics data in detail but does enable users to link a Phenopacket to files representing data from high-throughput screening techniques or to denote individual variants in several formats (see section on VRS and VRSATILE, below). ${ }^{45}$ The Phenopacket schema integrates the GA4GH Variant Representation Specification and is designed to be interoperable with other GA4GH standards including those for pedigree data. Figure 1 highlights the key building blocks of the Phenopacket schema, some of which are described in further detail below. 
medRxiv preprint doi: https://doi.org/10.1101/2021.11.27.21266944; this version posted November 30, 2021. The copyright holder for this preprint (which was not certified by peer review) is the author/funder, who has granted medRxiv a license to display the preprint in perpetuity.

It is made available under a CC-BY 4.0 International license .

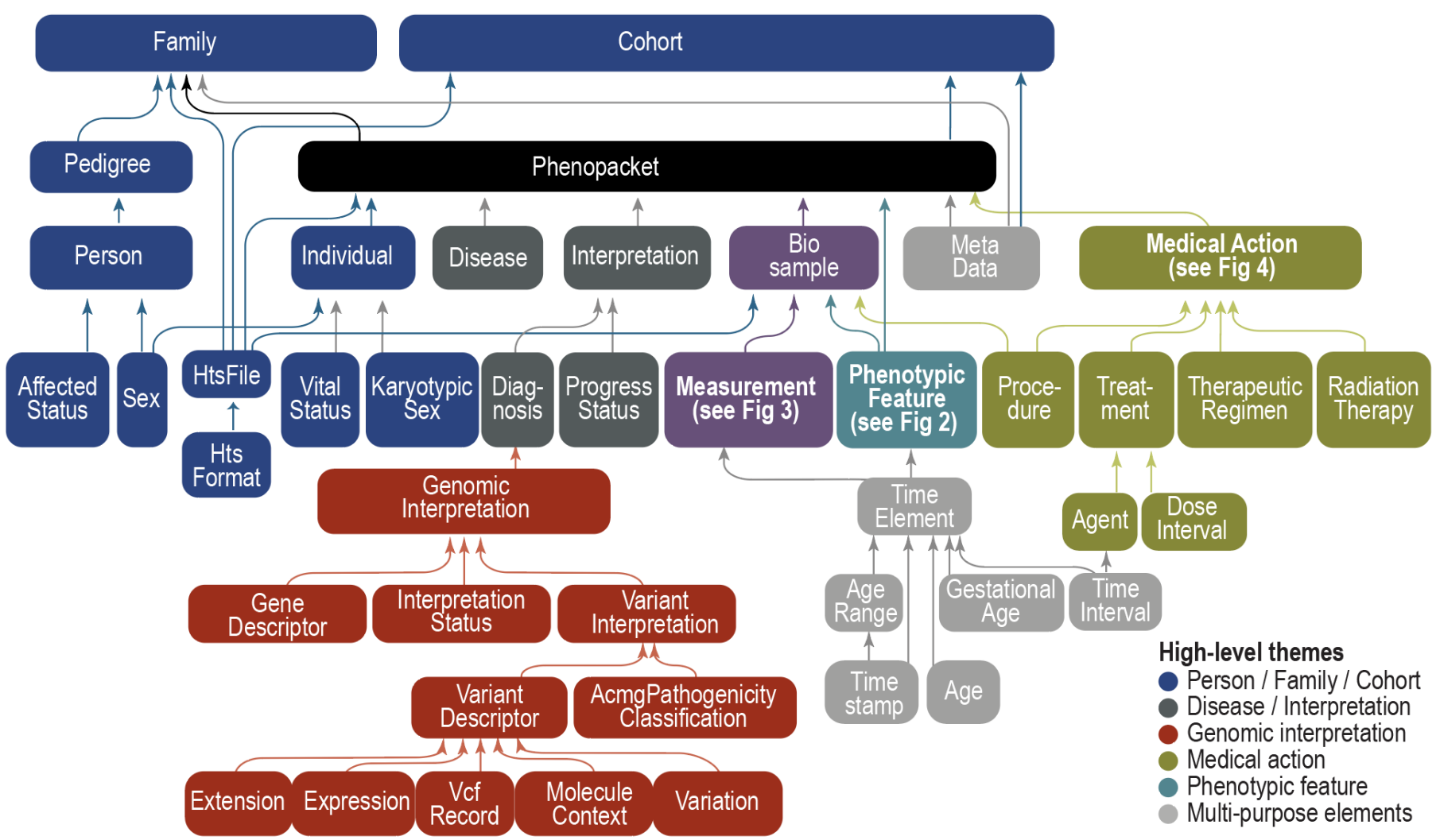

Figure 1. Phenopacket schema overview. The GA4GH Phenopacket schema consists of several optional elements, each of which contains information about a certain topic such as phenotype, variant, pedigree, etc. One element can contain other elements, which allows a hierarchical representation of data. For instance, the Phenopacket contains elements of type Individual, PhenotypicFeature, Biosample, and so on. Individual elements can therefore be regarded as building blocks that are combined to create larger structures. Colors represent the major themes of elements within the schema. See Figures 2, 3, and 4 for specifications of the PhenotypicFeature, Measurement, and Medical Action elements.

\section{Elements of a Phenopacket}

In the sections that follow, we describe a selection of the most important Phenopacket elements (see Figure 1). For the complete schema and detailed documentation for each element, please refer to the online documentation (see Web Resources).

\section{PhenotypicFeature}

The PhenotypicFeature (Fig. 2) is the central element of the Phenopacket schema. A PhenotypicFeature can be used to describe each phenotypic feature (often, but not necessarily, clinical abnormalities) including signs and symptoms, laboratory findings, histopathology findings, imaging, electrophysiological results, etc., along with modifier and qualifier concepts. Sources of phenotypes include physicians, laboratories, and patients; as wearables become more ubiquitous and more capable, these too will be important sources of phenotypes and context. Each phenotypic feature is described using an ontology term. While the Phenopacket schema does not mandate which ontology to use, there are recommendations, such as the HPO for rare diseases and the National Cancer Institute Thesaurus (NCIT) for transmission of information about a cancer specimen such as pathological staging or more detailed information about histology or tumor markers. ${ }^{46}$ One can indicate whether a certain abnormality was excluded during the diagnostic process (e.g., whether a morphological cardiac defect was excluded by echocardiography), or use other optional HPO terms to denote the severity of the PhenotypicFeature or other modifiers that describe the frequency (e.g., number of occurrences of seizures per week), laterality (e.g., unilateral) or other pattern of a certain phenotypic feature in the patient being described. Finally, the onset (and if applicable the resolution) of specific features can be indicated. Further information on this and other elements is available in the online documentation (See Web Resources). 
medRxiv preprint doi: https://doi.org/10.1101/2021.11.27.21266944; this version posted November 30, 2021. The copyright holder for this preprint (which was not certified by peer review) is the author/funder, who has granted medRxiv a license to display the preprint in perpetuity.

It is made available under a CC-BY 4.0 International license.

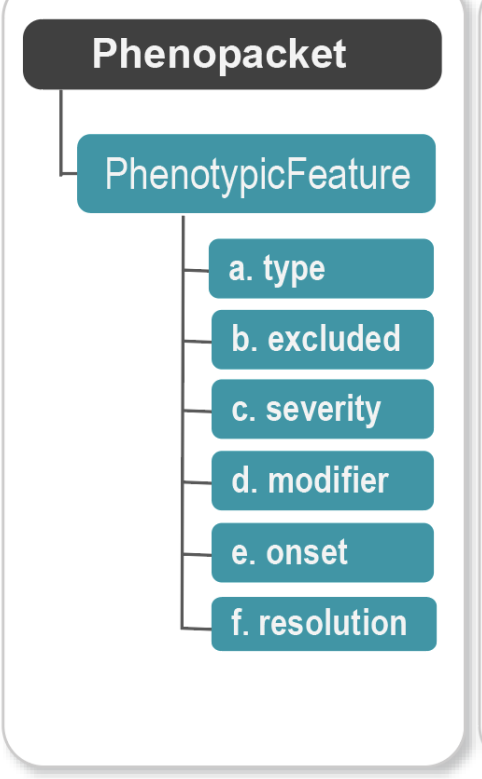

\section{Example}

\section{PhenotypicFeature}

type:

id: $\quad$ "HP:0012469"

label: "Infantile spasms"

severity:

id: "HP:0012828"

label: "Severe"

modifiers:

id: "HP:0025305"

label: "Quotidian"

onset:

id: "HP:0003593"

label: "Infantile onset"

resolution:

age:

is08601duration: "P4Y2M"

Figure 2. PhenotypicFeatures in a Phenopacket. A Phenopacket can contain information about an arbitrary number of phenotypic features observed in a single individual, each encoded using a PhenotypicFeature element. For medical use cases the subject will generally be a patient or a proband of a study, and the phenotypes will be abnormalities described by an ontology such as the HPO. Each phenotypic feature is defined by an HPO term (a), which is qualified as either present or absent (excluded) (b), with possible severity (c), modifiers (d), onset (e), and resolution (f). The example in the right panel shows a phenotypic feature, severe daily infantile spasms, which first occurred in infancy and resolved at age 4 years and 2 months, in a child without global developmental delay.

\section{Measurement}

The Measurement element is used to capture quantitative, ordinal (e.g., absent/present), or categorical measurements. For applications such as phenotype-driven genomic diagnostics of rare disease, qualitative representations of phenotypic abnormalities are appropriate, e.g., 'Thrombocytopenia' (HP:0001873). For other use cases, such as following the development of some parameter over time, the original quantitative values may be preferable, and can be represented using the Measurement element; for instance, 17,000 platelets per microliter. Measurement objects can be used to represent normal or abnormal measurements or to represent complex measurements with multiple components such as blood pressure.

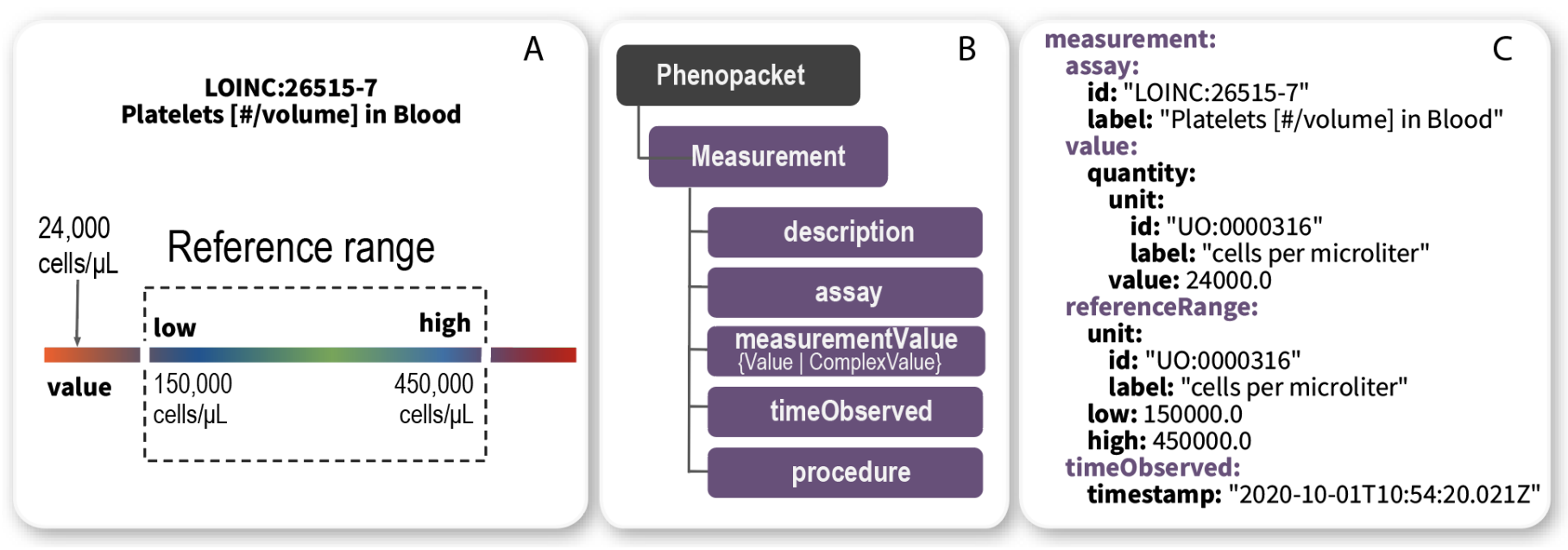

Figure 3. Example measurement of platelet count. Panel A: graphical representation of abnormal laboratory value outside of reference range. Panel B: relevant Phenopacket hierarchy. Panel C: part of a Measurement representing an abnormally low value for thrombocyte count. The reference range represents the range that was applied in the specific investigation and thus may reflect age or sex-specific values for some analytes. 
medRxiv preprint doi: https://doi.org/10.1101/2021.11.27.21266944; this version posted November 30, 2021. The copyright holder for this preprint (which was not certified by peer review) is the author/funder, who has granted medRxiv a license to display the preprint in perpetuity.

It is made available under a CC-BY 4.0 International license.

\section{Biosample}

A Biosample is a description of biological material obtained from the individual represented in the Phenopacket and used for phenotypic, genotypic, or other -omics analysis. For instance, a Biosample can represent a biopsy of an 'infiltrating urothelial carcinoma' (NCIT:C39853) taken from the urinary bladder wall (UBERON:0001256) by 'radical cystoprostatectomy' (NCIT:C51899) and found to be 'stage II' (NCIT:C28054) for which genome sequencing was performed, with a link to the VCF sequence variant file. If desired, Biosample can represent samples derived from other Biosamples, e.g., an RNA sample derived from a tumor. Interpretations of the genomic findings in a Biosample, if available, are represented in Interpretation elements (see below).

\section{MedicalAction including Treatment}

For cancer, ${ }^{47}$ infectious disease ${ }^{48}$, rare disease,${ }^{49}$ and many categories of common disease, ${ }^{50}$ precision-medicine approaches to treatment based on classification by genetic variants are rapidly gaining in importance. Consequently, clinical decision-making needs to integrate genomic research findings with structured information about treatments. The GA4GH Phenopacket schema includes a hierarchical representation of medical actions including medications, procedures, and other actions taken for clinical management. The Treatment element represents administration of a pharmaceutical agent, broadly defined as prescription and over-the-counter medicines, vaccines, and other therapeutic agents such as monoclonal antibodies or CAR T-cell-therapy.

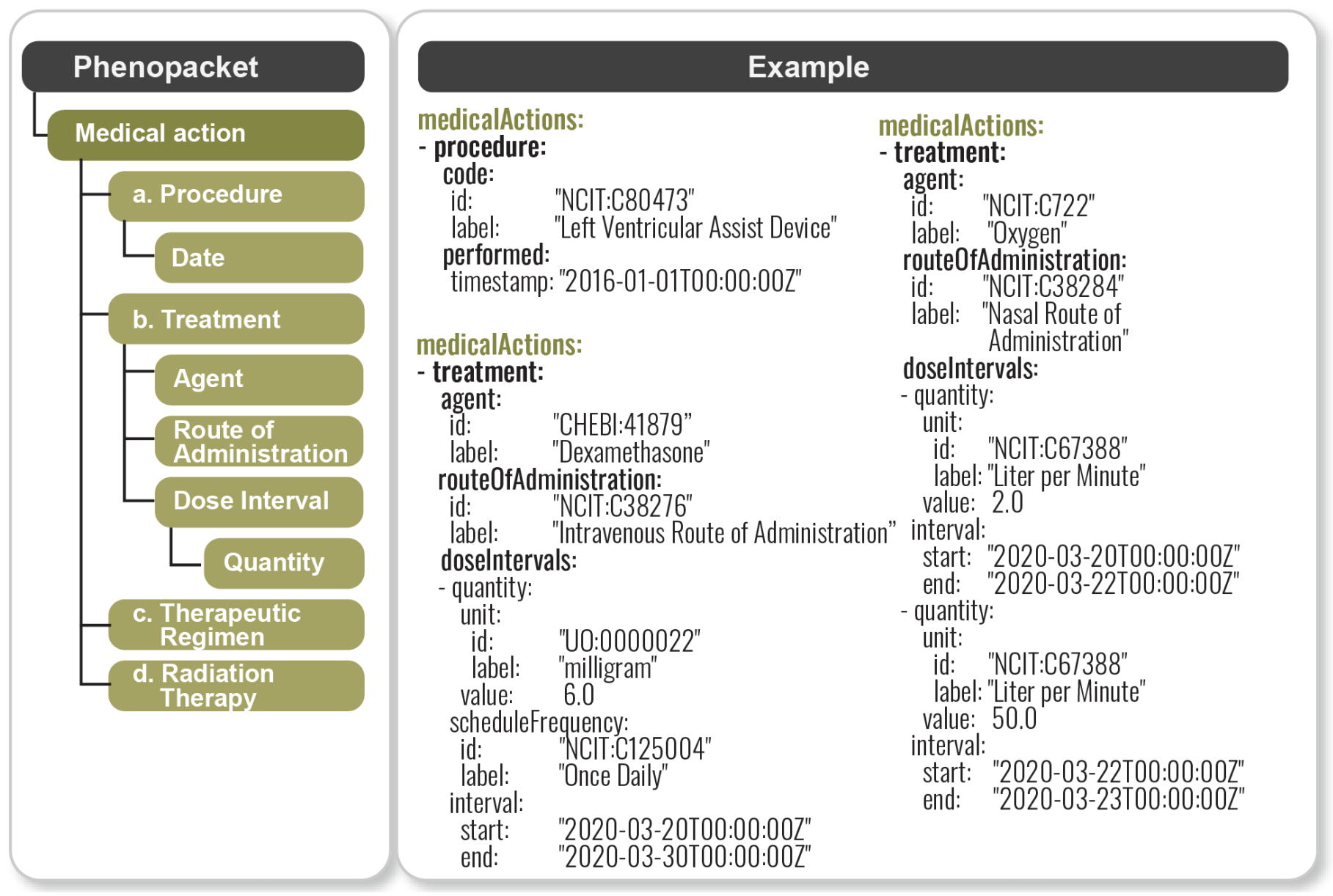

Figure 4. Phenopacket MedicalAction representation. Left panel: components of a MedicalAction in a Phenopacket. Right panel: example using COVID medical actions: implantation of a left ventricular assist device in 2016 (this information from the past medical history is important as it represents a risk factor for severe COVID-19 infection), intravenous administration of dexamethasone, and provision of oxygen by nasal cannula at an initial dose of 2 liters per minute that was later increased to 50 liters a minute. In general, a MedicalAction consists of one of the four options (Procedure, Treatment, TherapeuticRegimen, or RadiationTherapy). A Phenopacket can include an arbitrary number of medical actions. 
medRxiv preprint doi: https://doi.org/10.1101/2021.11.27.21266944; this version posted November 30, 2021. The copyright holder for this preprint (which was not certified by peer review) is the author/funder, who has granted medRxiv a license to display the preprint in perpetuity.

It is made available under a CC-BY 4.0 International license .

\section{Interpretation}

A Phenopacket can contain one or more Interpretation elements that specify interpretations of genomic findings. For instance, a report from a diagnostic laboratory about a variant interpreted to be causal for a certain Mendelian disease may be represented as an Interpretation element, as can a report of a potentially actionable somatic variant for which a targeted cancer therapy is available.

As a GA4GH standard, the Phenopacket schema integrates with and leverages other GA4GH standards when applicable. Subsequent to the release of the Phenopacket schema v1, the GA4GH Genomic Knowledge Standards Work Stream developed a precise and extensible standard for the representation of genomic variants: the Variation Representation Specification (VRS, pronounced "verse"). The Phenopacket schema v2 leverages the computational precision of VRS while maintaining the flexibility of describing variation using human-readable variant description formats (such as SPDI ${ }^{51}$ or $\mathrm{HGVS}^{45}$ ) through collaborative development and adoption of the VRS Added Tools for Interoperable Loquacious Exchange (VRSATILE, pronounced "versatile"; see Web Resources).

VRSATILE provides two primary object classes that are used in the Phenopacket schema: the VariationDescriptor and the GeneDescriptor. These descriptor classes allow for the extension of computationally precise concepts (e.g., VRS alleles, HGNC gene identifiers ${ }^{52}$ ) with common additional attributes for systems to describe these concepts (e.g., identifier cross-references, HGVS descriptions, gene symbols, and informative contexts such as variant zygosity). This collaborative framework bridges existing variant representation formats and implementations to the more computationally precise concepts enabled by VRS.

\section{Discussion}

The VCF standard for storing genotyping data allowed a wide range of research groups to write software for analyzing such data. ${ }^{1}$ The GA4GH Phenopacket schema aspires to be similarly transformative in the landscape of genome analysis using phenotype data.

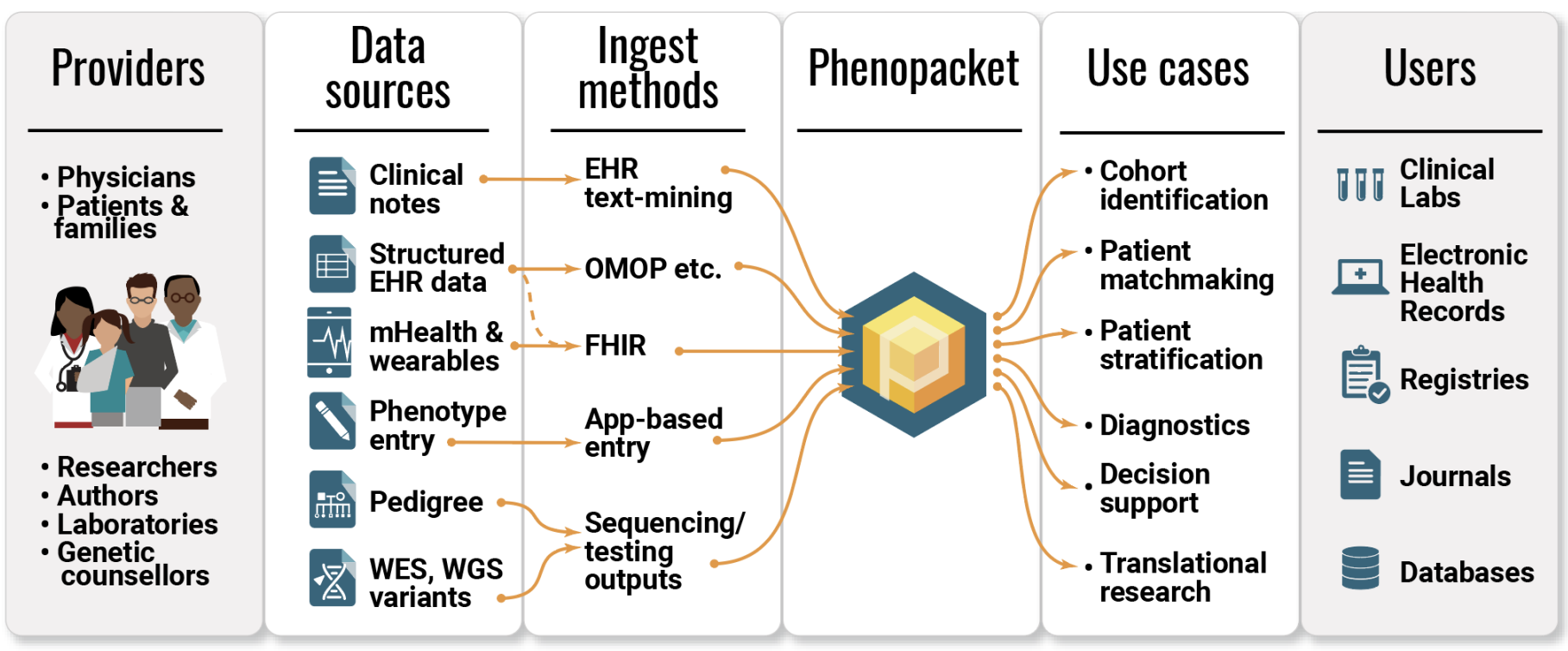

Figure 5. Phenotype data exchange in the biomedical ecosystem. Multiple providers of phenotypic data include patients and clinicians, via a variety of mechanisms including mHealth and the EHR. The Phenopacket schema acts as a common model that can capture data from many sources with a unified software representation and in turn can be used by multiple receivers of the phenotypic information, including journals, databases, registries, clinical laboratories. Phenopackets can support diverse users and use cases, including patient matchmaking services, diagnostics, and cohort identification. 


\section{Interoperability and integration of Phenopackets schema}

\section{Pedigree standards}

Unambiguous and computable pedigree information is critical for performing family-based genomic analysis. Pedigree data is currently represented in heterogeneous formats, such as custom JSON formats that can challenge interoperability, and lowest-common-denominator formats like PED (pedigree format), which are often limited in the representation of more complex families. The Phenopacket schema currently represents pedigree information using a simple transformation of the common PED format, which allows for describing the basic parent-child relationships necessary for family-based genetic analysis. Use cases that require richer information, such as clinical pedigrees, patient-reported family history data, and risk assessment for hereditary cancer, will be supported by integrating the GA4GH Pedigree Standard currently in development.

\section{GA4GH Beacon}

While the initial GA4GH Beacon standard empowered federated search for specific genomic variants, ${ }^{53}$ recent and upcoming versions of Beacon extend its scope through the use of standardized phenotypic data in both queries and data transfer. Beacon v2 has implemented a flexible filters extension for specifying additional query parameters that use ontology terms specified through CURIEs, in line with the Phenopacket schema. For data delivery, in contrast to the Boolean (true/false) responses provided through the original Beacon protocol, Beacon v2 allows for rich data delivery with response content described in default or external schemas. Although the v2 default schemas already track the Phenopacket schema to a large extent (e.g., Biosample), Beacon providers can decide to directly deliver Phenopacket responses to Beacon queries. Here, the greater sensitivity of richer data to be transferred through federated Beacon networks adds emphasis to the careful use of the tiered access control to increase security.

\section{EHR standards}

Medical coding systems and clinical exchange standards have not to date included rich phenotypic descriptions, as they are largely focused on supporting billing and clinical encounter documentation, rather than the documenting and sharing of the biologically relevant phenotypic information needed for computational use, mechanism discovery, and precision classification. From a clinical perspective, the integration of a standard for phenotypic description and exchange into and out of EHRs would improve disease diagnosis and management, especially for genomic health and precision medicine applications.

HL7 FHIR is a healthcare information exchange standard for EHR data. ${ }^{54} \mathrm{FHIR}$ is being implemented in EHR systems worldwide, and provides the opportunity to deploy a more robust phenotypic representation in the context of recording the clinical encounter natively within the EHR. The GA4GH Clinical and Phenotypic Data Capture Work Stream has developed a FHIR Implementation Guide (IG) that focuses on elements of the Phenopacket schema that are most commonly used for rare-disease genomic diagnostics (see Web Resources). Although the GA4GH Phenopacket was not designed to explicitly mirror existing data schemas for cancer, it was designed to cover the majority of data elements in the mCODE FHIR IG ${ }^{55}$ and the International Cancer Genome Consortium ARGO Data dictionary ${ }^{56}$ and we envision that multiple different FHIR resources such as mCODE can be exported using the GA4GH Phenopacket schema, enabling software to be written that will take advantage of a unified data format. The Phenopacket FHIR IG is being further developed under the HL7 FHIR Vulcan program. ${ }^{57}$

The GA4GH has committed to coordinate its activities and future roadmaps with those of other standards development organizations (SDOs), including International Organization for Standardization (ISO) Technical Subcommittee for Genomics Informatics (ISO/TC215/SC1) and HL7 Clinical Genomics (CG). The ISO version of the Phenopacket schema documentation, titled "Genomics Informatics - Phenopackets: A Format for Phenotypic Data Exchange", has been developed with input from both ISO and GA4GH stakeholders and aligned with the GA4GH Phenopacket schema. The final ISO balloting has been initiated, with publication planned for Spring 2022. This work will increase the availability of standardized phenotypic 
medRxiv preprint doi: https://doi.org/10.1101/2021.11.27.21266944; this version posted November 30, 2021. The copyright holder for this preprint (which was not certified by peer review) is the author/funder, who has granted medRxiv a license to display the preprint in perpetuity.

It is made available under a CC-BY 4.0 International license .

information and expand the collection of use cases to develop a standard relevant to genomics communities internationally.

\section{Applications of the Phenopacket schema}

The Phenopacket schema was designed to support a number of use cases. Many of these use cases have been implemented and tested in the community, particularly in the field of rare disease diagnostics and biobanking, while others, such as EHR integration, are in the process of being implemented. These use cases and implementations are discussed below.

\section{Rare-disease diagnostics}

Over 10,000 rare diseases have been identified to date ${ }^{58}$, collectively affecting between $3.5 \%$ and $8 \%$ of the population, ${ }^{59,60}$ yet many patients experience a long diagnostic odyssey of 5-7 years. ${ }^{44,58}$ To date, each of the numerous tools for phenotype-driven genomic diagnostic support have used bespoke input formats for the VCF file representing variants called from exome or genome sequencing, phenotypic data (generally in the form of a list of HPO terms), and information about the pedigree. The Phenopacket provides a standard input format for these tools that will simplify computational analysis pipelines, especially if the steps in the pipeline include a comparison of the results of multiple tools. Exomiser, ${ }^{29}$ LIRICAL, ${ }^{61}$ Phen2Gene, ${ }^{62}$ and CADA $^{63}$ can take Phenopackets as input files, and other analysis tools will soon accept phenotype data in Phenopacket format.

Multiple upstream data collection and management tools already support exporting patient profiles as phenopackets for downstream analysis and data sharing, including PhenoTips ${ }^{64}$ RD-Connect Genome-Phenome Analysis Platform (GPAP), Patient Archive in Australia and IRUD Exchange in Japan. PhenoTips can generate Phenopackets from patient or family records through a user interface or REST APIs, and includes de-identified demographic data, clinical phenotype, diagnoses, curated genetic findings, and pedigree data. Multiple projects, including the Children's Mercy Research Institute's Genomic Answers for Kids initiative, have adopted Phenopackets to help standardize data integration between PhenoTips and other systems. Projects such as EU funded Solve-RD and the European Joint Programme on Rare Diseases (EJP-RD) can generate Phenopackets for the data included in GPAP, which aims to facilitate diagnosis and novel gene discovery for clinical researchers. ${ }^{65}$ Phenopackets are used in Solve-RD to share phenotypic and other relevant clinical or genetic information (e.g., candidate or causative variants) between the consortium members, and are also deposited along the genomics data at the European Genome-phenome Archive (EGA) for long-term archival and controlled access. Besides being a successful instrument for data import/export between the project's databases, Phenopackets have also proved to be useful for data analysis, such as clustering patients based on their phenotypic similarity. Solve-RD has so far generated phenopackets from 11,349 individuals; this number is expected to increase soon to over $19,000.66$

\section{European Joint Programme on Rare Diseases (EJP RD)}

The EJP RD is developing a platform for federated discovery across rare diseases following the 'data visiting' strategy. ${ }^{67,68}$ The EJP RD is a GA4GH driver project, and several activities are making use of Phenopackets. On the topic of patient registries, the 24 involved European Reference Networks (ERNs) are working on applying the FAIR principles ${ }^{41}$ for the virtual data integration of different relevant resources in the platform. A FAIRification toolkit for the data stewards is under development to provide ready to use standards, where the Phenopacket schema is one of the planned clinical standards to be implemented. To improve the interoperability of Phenopackets within the FAIR platform, the project is providing Semantic Phenopackets based on Semantic Web standards and ontologies from the Open Biological and Biomedical Ontology (OBO) Foundry. ${ }^{69,70}$ The first release of this ontological modelling work is driven by the CAKUT (Congenital Anomalies of the Kidneys and Urinary Tract) rare disease registries use case ${ }^{71}$ Although at an early stage, the ability to seamlessly aggregate and integrate data from multiple registries or databases has huge potential benefits in terms of increasing the size of patient numbers for epidemiological analysis and facilitating the integration of distributed patient data into semantic knowledge graphs. To achieve full interoperability of ERNs rare disease patient registries with Phenopackets, the Semantic Phenopackets 
medRxiv preprint doi: https://doi.org/10.1101/2021.11.27.21266944; this version posted November 30, 2021. The copyright holder for this preprint (which was not certified by peer review) is the author/funder, who has granted medRxiv a license to display the preprint in perpetuity.

It is made available under a CC-BY 4.0 International license .

developed for the EJP RD virtual research platform will be further extended to include a full representation for all the elements of the GA4GH standard.

\section{BioSamples and the European Genome-phenome Archive (EGA)}

The BioSamples database at EMBL-EBI provides a central hub for sample metadata storage and linkage to other EMBL-EBI resources. ${ }^{72}$ BioSamples links to $\mathrm{EGA}^{73}$ and has done additional curation of human disease samples targeted to supporting Phenopackets. Every sample in BioSamples (over 19.5 million as of November 2021) exposes a Phenopacket record. Users can, for example, get a phenopacket for every single COVID-19 sample (see URLs).

\section{Biobanks}

In Japan, the Agency for Medical Research and Development (AMED) nation-wide biobank network has been developed to connect 12 major biobanks including Biobank Japan, Tohoku Medical Megabank project, and National Center Biobank Network. Together, the network stores over 855,181 biospecimens and 203,741 genomic data by 421,861 donors that are made available through a common query system exposing all phenotype data as phenopackets for downloading.

\section{Journals}

Published case reports contain data of immense value for characterizing and quantifying both new and existing conditions. However, these data are usually inaccessible for computational use, because they are represented in free-text or tabular formats designed for human readability. Even if the data are labeled using standardized ontology terms, there is no standardized format that can be used to disseminate the researchers' findings to the wider community. We envision that authors of articles that include descriptions of patients (case reports) could submit representations of the patients as Phenopackets at the time of manuscript submission to journals. This model is analogous to the structural biology field, where biological three-dimensional structures are required to be deposited with the Worldwide PDB (wwPDB) organization before publication. The wwPDB remains responsible for curating, storing and making structure data accessible, which maximizes the impact and utility of any work, both to the scientific community and from a funding perspective. Like with the structural biology community, a broader solution to the problem of providing access to structured phenotypic data in journal articles would be a collaboration between journals and databases, such as the EGA and the BioSamples database, to provide accessions and help authors deposit their data in a FAIR way.

\section{Patients and registries}

From the patient perspective, improved phenotype data sharing offers individuals and families affected by a disease the opportunity to share readily observable data through patient-centered phenotyping approaches. The recent translation of the clinically focused HPO into layperson terms ${ }^{52}$ and ongoing Indigenous language translations of the HPO under the Lyfe Languages initiative will facilitate patient-driven phenotyping and subsequent matching and discovery of additional patients. The ability to use Phenopackets is also incorporated in the new architecture of the Western Australian (WA) Register of Developmental Anomalies, which includes two registers, the WA Birth Defects Register and the WA Cerebral Palsy Register, and is connected to multiple health data sets and is part of the data linkage ecosystem in the WA Health Department.

\section{Configuring Phenopackets}

Because of the broad range of intended use cases and the large number of terminologies and ontologies in use by different communities, the Phenopacket schema is intentionally flexible with respect to which elements are required and which terminologies or ontologies must be used. Nonetheless, a given hospital, project, or research consortium may wish to apply different constraints. For instance, a Mendelian genetics consortium might stipulate the use of HPO terms to describe phenotypic abnormalities, and a cancer genomics consortium might require that each Phenopacket have a biosample for a tumor biopsy in which $\mathrm{NCIT}$ terms are used to describe the phenotypic features and other data. 
medRxiv preprint doi: https://doi.org/10.1101/2021.11.27.21266944; this version posted November 30, 2021. The copyright holder for this preprint (which was not certified by peer review) is the author/funder, who has granted medRxiv a license to display the preprint in perpetuity.

It is made available under a CC-BY 4.0 International license .

The Phenopacket schema itself has minimal requirements and most of the top-level fields are optional. The requirements are intended to enforce that elements have sufficient data to be unambiguous without requiring information that may not always be available. For instance, if the Quantity element is used, it must have the unit and value fields, but the referenceRange field is optional. The requirements for each element are defined in the online documentation. We have implemented a Java library and command-line application that validates Phenopackets using a JSON schema to enforce these constraints. The library, phenopacket-validator (see Web Resources), enables users to specify additional project- or consortium-specific constraints and to enforce validity of ontology terms used (examples are provided in the GitHub repository).

\section{Outlook}

Our goal is to provide a computable, well-structured representation of an individual's medically relevant data to serve a wide community of patients and clinicians, scientists, and software developers. A schema analogous to the VCF standard for genetic variation has been lacking for clinical data such as phenotypic features, disease diagnoses, and treatments. We have therefore developed the Phenopacket schema within the broad community of GA4GH to fill this need. Just as VCF is not currently able to represent all types of genomic variation, the Phenopacket schema does not cover all potentially relevant types of clinical information. The Phenopacket schema was designed to be easily integrated with other schemas that use the protobuf or JSON frameworks, and we envision future work to integrate the Phenopacket schema with standards to represent other areas including population background ('race' or 'ethnicity'), social determinants of health, and environmental exposures.

Software has become an essential resource for genomic medicine. A common experience of software developers is that a substantial amount of time is spent on reformatting data to correspond with the input format demanded by analysis software, or in the development of bespoke data schemes for new research projects. We hope that the Phenopacket schema will encourage the development of a collection of software for the analysis of genomic data in the context of clinical information that will accelerate innovation and discovery.

While the need for standardized computable and comparable clinical and genomic information is the basis for the development and anticipated implementation of Phenopackets, this does not replace a clear, concise, and nuanced narrative case report that describes the patient and the course of the disease over time. We strongly encourage the continued inclusion of case reports in addition to phenopackets in future publications.

Genomic data will become ever more important in translational research and clinical care in the coming years and decades. The Phenopacket schema represents a standard for capturing clinical data and integrating it with genomic data that will help to obtain the maximal utility of this data for understanding disease and developing precision medicine approaches to therapy.

\section{Web Resources:}

\section{Core Phenopacket resources}

Phenopacket schema source code: https://github.com/phenopackets/phenopacket-schema

Phenopacket schema documentation: https://phenopacket-schema.readthedocs.io/

Phenopacket validator: https://github.com/phenopackets/phenopacket-validator

Phenopacket tools: https://github.com/phenopackets/phenopacket-tools

\section{Related standards}

GA4GH Beacon project: https://beacon-project.io/ 
medRxiv preprint doi: https://doi.org/10.1101/2021.11.27.21266944; this version posted November 30, 2021. The copyright holder for this preprint (which was not certified by peer review) is the author/funder, who has granted medRxiv a license to display the preprint in perpetuity.

It is made available under a CC-BY 4.0 International license .

GA4GH Phenopacket FHIR implementation guide: https://github.com/phenopackets/core-ig

GA4GH Pedigree standard: https://github.com/GA4GH-Pedigree-Standard/pedigree

GA4GH Variation Representation Specification (VRS): vrs.ga4gh.org

VRS Added Tools for Interoperable Loquacious Exchange (VRSATILE): vrsatile.readthedocs.io

Phenopacket RDF model: https://github.com/LUMC-BioSemantics/phenopackets-rdf-schema/wiki

Genomics Informatics - Phenopackets: A Format for Phenotypic Data Exchange (ISO): https://www.iso.org/standard/79991.html

\section{Databases using Phenopackets}

The European Joint Programme on Rare Diseases (EJP RD): https://www.ejprarediseases.org/

BioSamples: https://www.ebi.ac.uk/biosamples (Search for NCBITaxon_2697049 to see BioSamples related to Severe acute respiratory syndrome coronavirus 2 or SAMN17024786 for a specific example).

AMED Biobank Network : http://biobank-network.jp/.

Contact: j.jacobsen@qmul.ac.uk; melissa@tislab.org; peter.robinson@jax.org

\section{Acknowledgements}

The authors gratefully acknowledge insight and feedback from Marian H. Adly, Orion J. Buske, Pier Luigi Buttigieg, Nour Gazzaz, Janine Lewis, Manuel Posada de la Paz and Maria Taboada

\section{Funding}

PNR was supported by NLM contract \#75N97019P00280, NIH NHGRI RM1HG010860, NIH OD R24OD011883, NIH NICHD 1R01HD103805-01. HH was supported by NIH OD R24OD011883. GIS was supported by ELIXIR, the research infrastructure for life-science data. CGC was supported by NIH NCATS U24TR002306. KCL was supported by NIH OD 5UM1OD023221. MB was supported by BioMedIT Network project of Swiss Institute of Bioinformatics (SIB) and Swiss Personalized Health Network (SPHN). AHW was supported by NIH NHGRI K99HG010157, NIH NHGRI R00HG010157. CJM, MAH, MCM-T, JAM, DD were supported by NIH NHGRI RM1HG010860, NIH OD R24OD011883. AM-J was supported by Australian Genomics. Australian Genomics is supported by the National Health and Medical Research Council (GNT1113531). DS, JOBJ were supported by NIH NHGRI RM1HG010860, NIH OD R24OD011883, NIH NICHD 1R01HD103805-01. MD was supported by NIH NHGRI U54HG004028, NIH NHGRI 5U01HG008473-03, NIH NCATS OT2TR003434-01S1U54HG008033-01. GSB was supported by Roy Hill Community Foundation, Angela Wright Bennett Foundation, McCusker Charitable Foundation, Borlaug Foundation, Stan Perron Charitable Foundation. LB was supported by NIH NHGRI U41HG006834 (Clinical Genome Resource). MC was supported by EMBL-EBI Core Funds and Wellcome Trust GA4GH award number 201535/Z/16/Z. AH was supported by NIH NHGRI 1U41HG006627, NIH NHGRI 1U54HG006542, NIH NHGRI 1RM1HG010860. PNS was supported by The Alan Turing Trust. NLH was supported by NIH NHGRI RM1HG010860, NIH OD R24OD011883, U.S. Department of Energy Contract DE-AC02-05CH11231. NP was supported by Moorfields Eye Charity. NQ-R was supported by EU Horizon 2020 research and innovation programme grant agreement 825575 (EJP-RD). OE was supported by NIH grants UL1TR002384, R01CA194547, P01CA214274 LLS SCOR grants 180078-01, 7021-20, Starr Cancer Consortium Grant I11-0027. HL was supported by CIHR Foundation Grant on Precision Health for Neuromuscular Diseases FDN-167281. RT was supported by CIHR postdoctoral fellowship award MFE-171275. LDS was supported by Genome Canada and NIH NHGRI U24HG011025. SO was supported 
medRxiv preprint doi: https://doi.org/10.1101/2021.11.27.21266944; this version posted November 30, 2021. The copyright holder for this preprint (which was not certified by peer review) is the author/funder, who has granted medRxiv a license to display the preprint in perpetuity.

It is made available under a CC-BY 4.0 International license .

by AMED. DP, LM, AP, SB, MR, RK were supported by EU Horizon 2020 research and innovation programme grant agreements 779257 (Solve-RD) and 825575 (EJP-RD). RRF was supported by NLM contract \#75N97019P00280.

\section{Contributions}

PNR contributed clinical data model expertise, clinical subject matter expertise, data analysis, data curation, data integration, data quality assurance, funding acquisition, manuscript drafting, critical revision of the manuscript for important intellectual content, software engineering, statistical analysis. JSB contributed clinical data model expertise, clinical subject matter expertise, critical revision of the manuscript for important intellectual content. $\mathrm{HH}$ collaborated on data integration, data quality assurance, funding acquisition, manuscript drafting, critical revision of the manuscript for important intellectual content, software engineering. PB is acknowledged for biological subject matter expertise. GIS collaborated on biological subject matter expertise, clinical subject matter expertise, data curation, data integration, database / information systems admin, funding acquisition, governance, marketing and communications, project evaluation. SK contributed clinical data model expertise, data integration. CGC contributed clinical data model expertise, funding acquisition, governance, critical revision of the manuscript for important intellectual content, project management. KCL contributed funding acquisition, critical revision of the manuscript for important intellectual content. PMK contributed biological subject matter expertise, clinical data model expertise, clinical subject matter expertise. MB contributed biological subject matter expertise, clinical data model expertise, database / information systems admin, manuscript drafting, project evaluation, software engineering. AHW contributed clinical data model expertise, data integration, manuscript drafting, software engineering. CFB collaborated on critical revision of the manuscript for important intellectual content. CJM contributed software engineering. AM-J contributed clinical data model expertise, software engineering. MT is acknowledged for . MP is acknowledged for biological subject matter expertise, clinical data model expertise, clinical subject matter expertise, data analysis, data curation, data integration, data quality assurance, data visualization, database / information systems admin, funding acquisition, governance, manuscript drafting, critical revision of the manuscript for important intellectual content, marketing and communications, project evaluation, project management, regulatory oversight / admin, software engineering, statistical analysis. DS contributed data analysis, data quality assurance, funding acquisition, project evaluation. ST contributed clinical data model expertise, clinical subject matter expertise, data integration. NAV contributed data curation. BPC collaborated on clinical data model expertise, clinical subject matter expertise, critical revision of the manuscript for important intellectual content. MD collaborated on biological subject matter expertise, clinical data model expertise, data integration, data quality assurance, manuscript drafting, critical revision of the manuscript for important intellectual content. HC collaborated on clinical subject matter expertise, data analysis, data curation, data integration, critical revision of the manuscript for important intellectual content. GSB contributed clinical subject matter expertise, funding acquisition, manuscript drafting, marketing and communications. LB collaborated on data analysis, data integration, software engineering. MC contributed biological subject matter expertise, clinical data model expertise, data curation, data integration, data quality assurance, data visualization, database / information systems admin, critical revision of the manuscript for important intellectual content, software engineering. TG contributed data integration, software engineering. VAK collaborated on biological subject matter expertise, clinical data model expertise, clinical subject matter expertise. PIS collaborated on clinical data model expertise, clinical subject matter expertise, data curation, data quality assurance, critical revision of the manuscript for important intellectual content. AH contributed clinical data model expertise, clinical subject matter expertise. PNS contributed clinical data model expertise, manuscript drafting, critical revision of the manuscript for important intellectual content. AZ collaborated on clinical data model expertise, clinical subject matter expertise, critical revision of the manuscript for important intellectual content. NG is acknowledged for critical revision of the manuscript for important intellectual content. MAH contributed biological subject matter expertise, clinical data model expertise, data curation, funding acquisition, governance, manuscript drafting, critical revision of the 
medRxiv preprint doi: https://doi.org/10.1101/2021.11.27.21266944; this version posted November 30, 2021. The copyright holder for this preprint (which was not certified by peer review) is the author/funder, who has granted medRxiv a license to display the preprint in perpetuity.

It is made available under a CC-BY 4.0 International license .

manuscript for important intellectual content, marketing and communications, project management. NLH collaborated on funding acquisition, governance, critical revision of the manuscript for important intellectual content, project management. NP contributed database / information systems admin, critical revision of the manuscript for important intellectual content. NQ-R contributed clinical data model expertise, manuscript drafting, critical revision of the manuscript for important intellectual content. MCM-T contributed governance, project evaluation, project management. CW contributed data integration. AK contributed clinical data model expertise, data analysis, governance, critical revision of the manuscript for important intellectual content. DPH collaborated on clinical data model expertise. OE contributed biological subject matter expertise, clinical subject matter expertise. HL collaborated on biological subject matter expertise, clinical subject matter expertise, funding acquisition, governance. RT collaborated on biological subject matter expertise, clinical data model expertise, clinical subject matter expertise, data curation, funding acquisition. ARM collaborated on clinical data model expertise. JL is acknowledged for biological subject matter expertise, manuscript drafting. JOBJ contributed clinical data model expertise, data analysis, data integration, data quality assurance, manuscript drafting, marketing and communications, project management, software engineering. RS contributed manuscript drafting, critical revision of the manuscript for important intellectual content, software engineering. OJB is acknowledged for critical revision of the manuscript for important intellectual content, marketing and communications. EMS contributed clinical data model expertise, clinical subject matter expertise, data curation, data integration, data quality assurance, critical revision of the manuscript for important intellectual content. MA collaborated on biological subject matter expertise, critical revision of the manuscript for important intellectual content. JS contributed data curation, critical revision of the manuscript for important intellectual content. MHA is acknowledged for clinical data model expertise, clinical subject matter expertise, data analysis, data integration, data quality assurance, database / information systems admin, governance, manuscript drafting, critical revision of the manuscript for important intellectual content, statistical analysis. LDS contributed manuscript drafting. JAM contributed data visualization, funding acquisition, manuscript drafting, critical revision of the manuscript for important intellectual content. DD contributed software engineering. MAG contributed data integration, software engineering. HL contributed biological subject matter expertise, critical revision of the manuscript for important intellectual content. SO contributed clinical data model expertise, manuscript drafting, project evaluation. TJC contributed clinical data model expertise, data analysis, data integration. AS contributed clinical data model expertise, data integration, software engineering. JLW contributed clinical subject matter expertise. DP contributed software engineering. LM contributed software engineering. AP contributed software engineering. SB contributed manuscript drafting, software engineering. MR contributed software engineering. RK contributed data integration, software engineering. BJL contributed clinical subject matter expertise. RRF contributed biological subject matter expertise, clinical data model expertise, data integration, funding acquisition, governance.

\section{Conflicts of interest}

SK is an employee of Ada Health $\mathrm{GmbH}$. DS is a consultant for Congenica Ltd. NP is a director of Phenopolis Ltd. OE is supported by Janssen, Johnson and Johnson, Volastra Therapeutics, AstraZeneca and Eli Lilly research grants. He is scientific advisor and equity holder in Freenome, Owkin, Volastra Therapeutics and One Three Biotech. ARM is an employee of Philips Research North America. JOBJ is a consultant for Congenica Ltd. OJB is an employee of PhenoTips. MA is an editor employed by Wiley. AS is an employee of Lifebit Biotech Ltd. 
medRxiv preprint doi: https://doi.org/10.1101/2021.11.27.21266944; this version posted November 30, 2021. The copyright holder for this preprint (which was not certified by peer review) is the author/funder, who has granted medRxiv a license to display the preprint in It is made available under a CC-BY 4.0 International license.

\section{References}

1. Danecek, P. et al. The variant call format and VCFtools. Bioinformatics 27, 2156-2158 (2011).

2. Richesson, R. \& Smerek, M. Electronic health records-based phenotyping. Rethinking clinical trials: $A$ living textbook of pragmatic clinical trials 2016, (2014).

3. Hripcsak, G. \& Albers, D. J. Next-generation phenotyping of electronic health records. J. Am. Med. Inform. Assoc. 20, 117-121 (2013).

4. Shivade, C. et al. A review of approaches to identifying patient phenotype cohorts using electronic health records. J. Am. Med. Inform. Assoc. 21, 221-230 (2014).

5. Wei, W.-Q. \& Denny, J. C. Extracting research-quality phenotypes from electronic health records to support precision medicine. Genome Med. 7, 41 (2015).

6. Richesson, R. L., Sun, J., Pathak, J., Kho, A. N. \& Denny, J. C. Clinical phenotyping in selected national networks: demonstrating the need for high-throughput, portable, and computational methods. Artif. Intell. Med. 71, 57-61 (2016).

7. Jayakumar, P. et al. Digital Phenotyping and Patient-Generated Health Data for Outcome Measurement in Surgical Care: A Scoping Review. J Pers Med 10, (2020).

8. Lenzi, A., Maranghi, M., Stilo, G. \& Velardi, P. The social phenotype: Extracting a patient-centered perspective of diabetes from health-related blogs. Artif. Intell. Med. 101, 101727 (2019).

9. Yamaguchi, A. \& Queralt-Rosinach, N. A proof-of-concept study of extracting patient histories for rare/intractable diseases from social media. Genomics Inform. 18, e17 (2020).

10. Haendel, M. A., Chute, C. G. \& Robinson, P. N. Classification, Ontology, and Precision Medicine. N. Engl. J. Med. 379, 1452-1462 (2018).

11. Robinson, P. N. Deep phenotyping for precision medicine. Hum. Mutat. 33, 777-780 (2012).

12. Weng, C., Shah, N. H. \& Hripcsak, G. Deep phenotyping: Embracing complexity and temporality-Towards scalability, portability, and interoperability. J. Biomed. Inform. 105, 103433 (2020).

13. Buske, O. J. et al. PhenomeCentral: a portal for phenotypic and genotypic matchmaking of patients with rare genetic diseases. Hum. Mutat. 36, 931-940 (2015).

14. Wright, C. F. et al. Genetic diagnosis of developmental disorders in the DDD study: a scalable analysis of genome-wide research data. Lancet 385, 1305-1314 (2015). 
medRxiv preprint doi: https://doi.org/10.1101/2021.11.27.21266944; this version posted November 30, 2021. The copyright holder for this preprint (which was not certified by peer review) is the author/funder, who has granted medRxiv a license to display the preprint in It is made available under a CC-BY 4.0 International license.

15. Firth, H. V., Wright, C. F. \& Study, D. The Deciphering Developmental Disorders (DDD) study. Dev. Med. Child Neurol. 53, 702-703 (2011).

16. Feenstra, I. et al. European Cytogeneticists Association Register of Unbalanced Chromosome Aberrations (ECARUCA); an online database for rare chromosome abnormalities. Eur. J. Med. Genet. 49, 279-291 (2006).

17. Bragin, E. et al. DECIPHER: database for the interpretation of phenotype-linked plausibly pathogenic sequence and copy-number variation. Nucleic Acids Res. 42, D993-D1000 (2014).

18. Marx, V. The DNA of a nation. Nature 524, 503-505 (2015).

19. Tifft, C. J. \& Adams, D. R. The National Institutes of Health undiagnosed diseases program. Curr. Opin. Pediatr. 26, 626-633 (2014).

20. Pontikos, N. et al. Phenopolis: an open platform for harmonization and analysis of genetic and phenotypic data. Bioinformatics 33, 2421-2423 (2017).

21. Savatt, J. M. et al. ClinGen's GenomeConnect registry enables patient-centered data sharing. Hum. Mutat. 39, 1668-1676 (2018).

22. Kirkpatrick, B. E. et al. GenomeConnect: matchmaking between patients, clinical laboratories, and researchers to improve genomic knowledge. Hum. Mutat. 36, 974-978 (2015).

23. Beaulieu, C. L. et al. FORGE Canada Consortium: outcomes of a 2-year national rare-disease gene-discovery project. Am. J. Hum. Genet. 94, 809-817 (2014).

24. Ramoni, R. B. et al. The Undiagnosed Diseases Network: Accelerating Discovery about Health and Disease. Am. J. Hum. Genet. 100, 185-192 (2017).

25. Thompson, R. et al. RD-Connect: an integrated platform connecting databases, registries, biobanks and clinical bioinformatics for rare disease research. J. Gen. Intern. Med. 29 Suppl 3, S780-7 (2014).

26. Köhler, S. et al. The Human Phenotype Ontology project: linking molecular biology and disease through phenotype data. Nucleic Acids Res. 42, D966-D974 (2014).

27. Köhler, S. et al. Expansion of the Human Phenotype Ontology (HPO) knowledge base and resources. Nucleic Acids Res. 47, D1018-D1027 (2019).

28. Köhler, S. et al. Clinical diagnostics in human genetics with semantic similarity searches in ontologies. Am. J. Hum. Genet. 85, 457-464 (2009). 
medRxiv preprint doi: https://doi.org/10.1101/2021.11.27.21266944; this version posted November 30, 2021. The copyright holder for this preprint (which was not certified by peer review) is the author/funder, who has granted medRxiv a license to display the preprint in It is made available under a CC-BY 4.0 International license.

29. Robinson, P. N. et al. Improved exome prioritization of disease genes through cross-species phenotype comparison. Genome Res. 24, 340-348 (2014).

30. Smedley, D. et al. A Whole-Genome Analysis Framework for Effective Identification of Pathogenic Regulatory Variants in Mendelian Disease. Am. J. Hum. Genet. 99, 595-606 (2016).

31. Smedley, D. et al. Next-generation diagnostics and disease-gene discovery with the Exomiser. Nat. Protoc. 10, 2004-2015 (2015).

32. Köhler, S. et al. The Human Phenotype Ontology in 2021. Nucleic Acids Res. 49, D1207-D1217 (2021).

33. Pendergrass, S. A. \& Crawford, D. C. Using Electronic Health Records To Generate Phenotypes For Research. Curr. Protoc. Hum. Genet. 100, e80 (2019).

34. Zuk, O. et al. Searching for missing heritability: designing rare variant association studies. Proc. Natl. Acad. Sci. U. S. A. 111, E455-64 (2014).

35. Beckmann, J. S. \& Lew, D. Reconciling evidence-based medicine and precision medicine in the era of big data: challenges and opportunities. Genome Med. 8, 134 (2016).

36. Krawitz, P., Buske, O., Zhu, N., Brudno, M. \& Robinson, P. N. The genomic birthday paradox: how much is enough? Hum. Mutat. 36, 989-997 (2015).

37. Friedman, C. et al. Toward a science of learning systems: a research agenda for the high-functioning Learning Health System. J. Am. Med. Inform. Assoc. 22, 43-50 (2015).

38. Friedman, C. P., Wong, A. K. \& Blumenthal, D. Achieving a nationwide learning health system. Sci. Transl. Med. 2, 57cm29 (2010).

39. Global Alliance for Genomics and Health. GENOMICS. A federated ecosystem for sharing genomic, clinical data. Science 352, 1278-1280 (2016).

40. Haendel, M. A. et al. A Census of Disease Ontologies. Annu. Rev. Biomed. Data Sci. 1, 305-331 (2018).

41. Wilkinson, M. D. et al. The FAIR Guiding Principles for scientific data management and stewardship. Sci Data 3, 160018 (2016).

42. Wilson, S. L. et al. Sharing biological data: why, when, and how. FEBS Lett. 595, 847-863 (2021).

43. Haendel, M. et al. Metrics to assess value of biomedical digital repositories: response to RFI 
medRxiv preprint doi: https://doi.org/10.1101/2021.11.27.21266944; this version posted November 30, 2021. The copyright holder for this preprint (which was not certified by peer review) is the author/funder, who has granted medRxiv a license to display the preprint in It is made available under a CC-BY 4.0 International license .

NOT-OD-16-133. Geneva: Zenodo (2016).

44. Rubinstein, Y. R. et al. The case for open science: rare diseases. Jamia Open doi:10.1093/jamiaopen/ooaa030.

45. den Dunnen, J. T. Describing Sequence Variants Using HGVS Nomenclature. Methods Mol. Biol. 1492, 243-251 (2017).

46. Sioutos, N. et al. $\mathrm{NCl}$ Thesaurus: a semantic model integrating cancer-related clinical and molecular information. J. Biomed. Inform. 40, 30-43 (2007).

47. Schmitz, R. et al. Genetics and Pathogenesis of Diffuse Large B-Cell Lymphoma. N. Engl. J. Med. 378, 1396-1407 (2018).

48. Pletz, M. W., Bauer, M. \& Brakhage, A. A. One step closer to precision medicine for infectious diseases. The Lancet infectious diseases vol. 19 564-565 (2019).

49. Anwar, S. \& Yokota, T. Golodirsen for Duchenne muscular dystrophy. Drugs Today 56, 491-504 (2020).

50. Bodkin, J. A. et al. Targeted Treatment of Individuals With Psychosis Carrying a Copy Number Variant Containing a Genomic Triplication of the Glycine Decarboxylase Gene. Biol. Psychiatry 86, 523-535 (2019).

51. Holmes, J. B., Moyer, E., Phan, L., Maglott, D. \& Kattman, B. SPDI: data model for variants and applications at NCBI. Bioinformatics 36, 1902-1907 (2020).

52. Tweedie, S. et al. Genenames.org: the HGNC and VGNC resources in 2021. Nucleic Acids Res. 49, D939-D946 (2021).

53. Fiume, M. et al. Federated discovery and sharing of genomic data using Beacons. Nat. Biotechnol. 37, 220-224 (2019).

54. Bender, D. \& Sartipi, K. HL7 FHIR: An Agile and RESTful approach to healthcare information exchange. in Proceedings of the 26th IEEE International Symposium on Computer-Based Medical Systems 326-331 (2013).

55. Osterman, T. J., Terry, M. \& Miller, R. S. Improving Cancer Data Interoperability: The Promise of the Minimal Common Oncology Data Elements (mCODE) Initiative. JCO Clin Cancer Inform 4, 993-1001 (2020). 
medRxiv preprint doi: https://doi.org/10.1101/2021.11.27.21266944; this version posted November 30, 2021. The copyright holder for this preprint (which was not certified by peer review) is the author/funder, who has granted medRxiv a license to display the preprint in It is made available under a CC-BY 4.0 International license.

56. Zhang, J. et al. The International Cancer Genome Consortium Data Portal. Nat. Biotechnol. 37, 367-369 (2019).

57. Health Level 7 (HL7). Vulcan Accelerator Home - Vulcan Accelerator - Confluence. https://confluence.hl7.org/display/VA/Vulcan+Accelerator+Home.

58. Haendel, M. et al. How many rare diseases are there? Nat. Rev. Drug Discov. (2019) doi:10.1038/d41573-019-00180-y.

59. Wakap, S. N. et al. Estimating cumulative point prevalence of rare diseases: analysis of the Orphanet database. Eur. J. Hum. Genet. 28, 165-173 (2019).

60. Laura Joszt, M. A. Not so rare: 300 million people worldwide affected by rare diseases. https://www.ajmc.com/view/not-so-rare-300-million-people-worldwide-affected-by-rare-diseases.

61. Robinson, P. N. et al. Interpretable Clinical Genomics with a Likelihood Ratio Paradigm. Genetic and Genomic Medicine (2020) doi:10.1101/2020.01.25.19014803.

62. Zhao, M. et al. Phen2Gene: rapid phenotype-driven gene prioritization for rare diseases. NAR Genom Bioinform 2, Iqaa032 (2020).

63. Peng, C. et al. CADA: phenotype-driven gene prioritization based on a case-enriched knowledge graph. NAR Genom Bioinform 3, (2021).

64. Girdea, M. et al. PhenoTips: Patient Phenotyping Software for Clinical and Research Use. Hum. Mutat. 34, 1057-1065 (2013).

65. Lochmüller, H. et al. RD-Connect, NeurOmics and EURenOmics: collaborative European initiative for rare diseases. Eur. J. Hum. Genet. 26, 778-785 (2018).

66. Zurek, B. et al. Solve-RD: systematic pan-European data sharing and collaborative analysis to solve rare diseases. Eur. J. Hum. Genet. 29, 1325-1331 (2021).

67. Kaliyaperumal, R. et al. Semantic modelling of Common Data Elements for Rare Disease registries, and a prototype workflow for their deployment over registry data. bioRxiv (2021) doi:10.1101/2021.07.27.21261169.

68. Beyan, O. et al. Distributed analytics on sensitive medical data: The Personal Health Train. Data Intellegence 2, 96-107 (2020).

69. Smith, B. et al. The OBO Foundry: coordinated evolution of ontologies to support biomedical data 
medRxiv preprint doi: https://doi.org/10.1101/2021.11.27.21266944; this version posted November 30, 2021. The copyright holder for this preprint (which was not certified by peer review) is the author/funder, who has granted medRxiv a license to display the preprint in It is made available under a CC-BY 4.0 International license.

integration. Nat. Biotechnol. 25, 1251-1255 (2007).

70. Bizer, C., Heath, T. \& Berners-Lee, T. Linked Data - The Story So Far. Int. J. Semant. Web Inf. Syst. 5, 1-22 (2009).

71. Klein, J. et al. Amniotic fluid peptides predict postnatal kidney survival in developmental kidney disease. Kidney Int. 99, 737-749 (2021).

72. Courtot, M. et al. BioSamples database: an updated sample metadata hub. Nucleic Acids Res. 47, D1172-D1178 (2019).

73. Lappalainen, I. et al. The European Genome-phenome Archive of human data consented for biomedical research. Nat. Genet. 47, 692-695 (2015). 